\title{
ANÁLISIS SOBRE LA PROPORCIONALIDAD DEL RITMO DE MIGRACIÓN CENTROAMERICANA A ESPAÑA, EN RELACIÓN AL ÍNDICE DE DESARROLLO HUMANO
}

Jesús David Argueta Moreno. Universidad Nacional Autónoma de Honduras, Facultad de Ciencias Económicas, Instituto de Investigaciones Económicas y Sociales (UNAH-FCE-IIES), Ciudad Universitaria, Edifício C2, Primer piso, Tel/Fax: 504-2239 1849

E-mail: jargueta@iies-unah.org

\section{RESUMEN:}

La presente investigación, de corte cualitativo, no experimental transversal, se enfoca en el estudio del fenómeno de la migración Centroamericana a España, desde la perspectiva de desarrollo social de estas comunidades en el periodo 1990-2011, a través del análisis de la interrelación, entre las variables: Tasa de Migración- Índice de Desarrollo Humano.

Asimismo, se evaluará cuál de los componentes, que conforman el Índice de Desarrollo Humano

(IDH), presentan una mayor incidencia sobre el mismo (sea el Indicador de Esperanza de Vida al Nacer, Índice de Educación o el Índice Producto Interno Bruto).

Para el desarrollo de la propuesta, se aborda un breve diagnóstico de las condiciones sociales y económicas del contexto centroamericano, que facilitara la comprensión y tendencias del IDH, en el tiempo.

Palabras clave: Desarrollo Humano, Desarrollo Social, Desarrollo Poblacional. 


\section{ANALYSIS ON THE PROPORTIONALITY OF THE RATE OF CENTRAL AMERICAN MIGRATION TO SPAIN, IN RELATION TO HUMAN DEVELOPMENT INDEX}

Jesús David Argueta Moreno. Universidad Nacional Autónoma de Honduras, Facultad de Ciencias Económicas, Instituto de Investigaciones Económicas y Sociales (UNAH-FCE-IIES), Ciudad Universitaria, Edifício C2, Primer piso, Tel/Fax: 504-2239 1849

E-mail: jargueta@iies-unah.org

\section{ABSTRACT:}

This qualitative, non-experimental research focuses on the study of the Central American migration phenomenon that impact on the European mainland (Spain). The investigation methodology, applies the social development perspective, as media to evaluate the exodus of the Central American communities to Spain, in the periods 1990-2011, embracing the analysis of the proportions between the variables: Migration Rate-Human Development Index.

Likewise, this study assesses the components that build up the Human Development Index (HDI), by identifying, which of these elements have a higher correlation on it's statistics.

The proposal also addresses a brief analysis of the social and economic conditions of the Central American context, to facilitate the understanding of the Human Development Index trends, in the 1990-2011 time line.

Keywords: Human Development, Social Development, Population Development. 


\section{OBJETIVO GENERAL:}

Evaluar la correlación entre las variables Tasa de Migración Centro Americana a España e Índice de Desarrollo Humano (IDH) en la región.

\section{OBJETIVOS ESPECÍFICOS:}

1. Evaluar la intensidad o nivel de dependencia entre las variables Índice de Desarrollo Humano (IDH) e Índice de Esperanza de Vida al Nacer (IEV) para el caso de Centro América.

2. Evaluar la intensidad o nivel de dependencia entre las variables Índice de Desarrollo Humano (IDH) e Índice de Educación (IE) para el caso de Centro América.

3. Evaluar la intensidad o nivel de dependencia entre las variables Índice de Desarrollo Humano (IDH) e Índice del Producto Interno Bruto (IPIB) para el caso de Centro América.

4. Determinar cuál de las variables, IPIB, IE o IEV, es la que presenta más incidencia en el Índice de Desarrollo Humano para Centro América.

\section{PREGUNTAS DE LA INVESTIGACIÓN:}

1. ¿A qué intensidad o nivel de dependencia, se genera la relación entre las variables el IDH y el IEV?

2. ¿A qué intensidad o nivel de dependencia, se genera la relación entre las variables el IDH y el IE?

3. ¿A qué intensidad o nivel de dependencia, se genera la relación entre las variables el IDH y el IPIB?

4. ¿Cuál de las variables, que conforman el IDH (IEV, IE e IPIB), presenta una mayor trascendencia sobre el mismo? 


\section{PLANTEAMIENTO DEL PROBLEMA}

\section{Antecedentes de la Investigación:}

La problemática hondureña, ha experimentado en los últimos años, una exponencial tendencia sobre los márgenes de pobreza, analfabetismo y salud. Según el "Panorama Social de América Latina", divulgado en el 2011, Honduras es uno de los países (en América Latina) con el índice de pobreza más elevado, con aproximadamente un $67.8 \%, 10$ puntos porcentuales arriba de Paraguay y 18 puntos porcentuales sobre El Salvador. ${ }^{1}$

“En Enero del 2010, con la idea de combatir las deficiencias, presentadas con anterioridad, El Congreso Nacional de Honduras aprobó la "Visión de País 2010 - 2038” y el "Plan de Nación 2010-2022", preparado por una comitiva nombrada por el mismo Congreso, con el supuesto de que pueda ejecutarse durante los siguientes siete períodos de Gobierno", amparados en los decretos 70-2002 y 43-2002, publicados en el Diario la Gaceta Nacional, previamente en el año $2002^{3}$.

De esta Política Macro de Gobierno, se acordó, fijar, lo siguiente:

a. Una Visión de País contentiva de Principios, Objetivos Nacionales y Metas de Prioridad Nacional para el período 2010-2038.

b. Un Plan de Nación que cubre una primera fase de la Visión de País (2010-2038) y que se conforma de lineamientos estratégicos, objetivos e indicadores que marcarán las progresivas responsabilidades de los próximos tres gobiernos en torno a la Visión de País.

c. Una matriz de 58 indicadores, que contiene los criterios cuantitativos a alcanzar y que permiten medir el avance del cumplimiento del Plan de Nación, según cada uno de los lineamientos estratégicos que lo componen, segmentados según al grado de avance esperado al final de cada periodo de gobierno.

d. Un anteproyecto de decreto para la creación del Sistema Nacional de Planeación que actúa como instrumento para la ejecución e institucionalización de la Visión de País y el Plan

$1 \mathrm{http} / /$ resistenciahonduras.net/index.php?option=com_content\&view=article\&id=4178:honduras-pais-con-mayor-pobreza-enamerica\&catid $=65$ :economia-social\&Itemid $=249$

$2 \mathrm{http} / /$ www.enlaceacademico.org/base-documental/biblioteca/documento/honduras-vision-de-pais-2010-2038-y-plan-denacion-2010-2022/

3 http://www.fosdeh.net/archivos/Ley \%20del \%20FRP Decreto\%20N70 \%2002.pdf 
de Nación y que debe contar, para su debida implementación, con un marco jurídico, una definición institucional y un esquema de funcionalidad operativa.

Esta medida de auxilio social y Financiero, se gesta como un recurso, para contrarrestar la exclusión social e inequidad social latente y manifiesta desde los distintos sectores y entornos del país, sin embargo, previo al abordaje del Plan de Nación 2010-2022, esta problemática se arrastra desde mucho tiempo atrás.

En el año 2000, el Estado de Honduras, decide adoptar como herramienta de primera mano, a la Estrategia para la Reducción de la Pobreza (ERP) como un compromiso y esfuerzo compartido entre el Gobierno (Dijkstra, 2007), en sus distintos ámbitos, y la sociedad hondureña, en lo atinente a su formulación y ejecución, seguimiento y evaluación. Este compromiso comienza como tal en el momento en que se incorpora como uno de los principales elementos del Plan Maestro de Reconstrucción y Transformación Nacional (PMRTN)4 ${ }^{4}$, espera sostenerse en el largo plazo, como política de Estado, en un marco de amplia participación de la sociedad civil, con el apoyo de la cooperación internacional.

¿Existen Programas de similar envergadura, en América Latina, que gesten de manera exitosa, propuestas similares al Plan de Nación y Visión de País, en Honduras?

Un intento similar se efectuó por Juscelino Kubitschek de Oliveira, quien en su administración como Presidencial Brasileño, desarrollo una propuesta de país (Terragno, 2010), la cual entre sus objetivos contemplaba lo siguiente:

Construcción de la gran red vial, que comunicara a todos los estados que constituyen el Brasil

- $\quad$ El Traslado de la capital de Rio de Janeiro a Brasilia.

- $\quad$ La Industrialización del País.

- $\quad$ La mitigación y reducción de la pobreza.

4 Grupo de Seguimiento a la Declaración de Estocolmo, http://www.gsdehonduras.org/index.php?option=com_ content $\&$ view $=$ frontpage $\&$ Itemid $=1$ 
Demostrando ser eficiente en la actualidad con un IDH de 0.718 , el cual, se pronostica, seguirá creciendo en la medida en que el país, intensifique sus políticas de industrialización en la Bioenergía, salud y alfabetización en el presente gobierno de la economista Dilma Rouseff5.

\section{Situación de la Problemática:}

Bajo la premisa de sustentar y resaltar, la importancia de la implementación del Plan de Nación 2010-2022, sobre la sociedad Hondureña y de forma efectiva hacer conciencia de la importancia de su rol en el porvenir Hondureño, es necesaria la creación de un diagnóstico puntual, que describa el ¿Por qué? del estancamiento social, a través de uno de los instrumentos de medición más eficaces en la actualidad, el Índice de Desarrollo Humano (IDH).

De forma paralela, el estudio del impacto del IDH (forma directa/indirecta), sobre el fenómeno de la migración Hondureña hacia el continente Europeo, particularmente, hacia España, es retomado por las políticas contempladas en el Plan de Nación y Visión de país (2010-2038), respecto a la mitigación

E\&A de la fuga de cerebros, mano de obra y talentos.

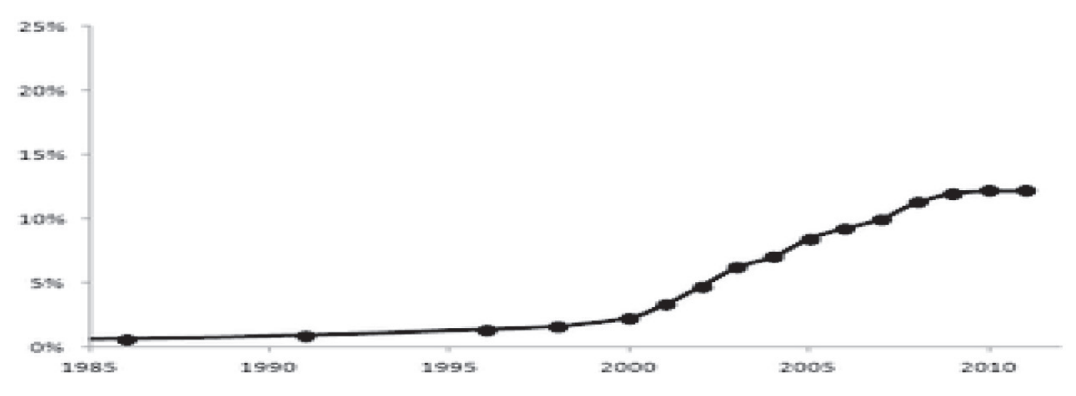

Gráfico No.1.

Inmigración Extranjera España:

Fuente: Eurostat: Población en Europa - 2005

Como se aprecia en el Gráfico No. 1, el análisis sobre aquellos extranjeros que migran, hacia España según las caídas en los patrones del IDH, es imperativo, para comprender la difícil situación que atraviesa el contexto latino americano, particularmente el hondureño, con la finalidad de rectificar y ajustar los fines perseguidos por el Plan de Nación, para encaminar y adoptar las medidas pertinentes.

5 PNUD, IDH Brasil, http://hdrstats.undp.org/es/paises/perfiles/BRA.html 


\section{JUSTIFICACIÓN DE LA INVESTIGACIÓN}

\section{Conveniencia:}

La investigación, se estima conveniente y oportuna, dado que esta propuesta de investigación, sustenta la necesidad de implementar y enfocarse en 6 de los 11 lineamientos estratégicos, contemplados en el Plan de Nación 2010-2022.

I. Desarrollo Sostenible de la Población.

II. Reducción de la Pobreza, Generación de Activos e igualdad de Oportunidades.

III. Educación y Cultura, como medios de emancipación social.

IV. Salud como fundamento para la mejora en las condiciones de vida.

V. Desarrollo Regional.

VI. Competitividad, Imagen País y Sectores Productivos.

Como un apartado que justifica la urgencia e inmediatez, de la aplicación de estas propuestas de desarrollo social a nivel local, así como regional.

De igual forma, esta investigación, pretende estimular la conciencia crítica del sector académico público, en el marco de la línea de Investigación del Instituto de Investigaciones Económicas y Sociales (IIES-UNAH) denominada “Análisis de la Cadena de Valor y Suministro: Oferta y Demanda de Profesionales de la Educación Superior"; y las 13 prioridades de investigación de la Dirección de Investigación Científica Universitaria (DICU) de la Universidad Nacional Autónoma de Honduras. ${ }^{7}$

\section{MARCO TEÓRICO DE LA PROPUESTA}

El presente análisis, gira en torno la Teoría de las proporciones de los elementos de Euclides de Alejandria(Cejas, 2002), adoptando al mismo, como modelo de línea base para el presente estudio, el cual evalua la proporcionalidad entre las caidas y alzas del IDH vrs. la tasa de migracion Hondureña hacia España.

6 www.iies-unah.org

7 https://www.unah.edu.hn/?cat=1133 
A través de la teoría formulada por el científico Euclides (300 a.C.), podremos analizar de manera cuantitativa, la proporcionalidad entre las variables Tasa de Migración-IDH, En primera instancia, demostrando el nivel de dependencia de sus variables y posteriormente, la sinergia de proporciones vinculadas al incremento de un componente y la caída de otro.

Cabe mencionar que el sistema creado por Euclides sistematizó todos los conocimientos de su época, ordenando las enseñanzas a su manera, validando los teoremas requeridos por su nueva ordenación lógica, basada en el método axiomático; partiendo de cinco axiomas, cuya verdad se considera evidente ${ }^{8}$. Los cuales son:

1.-Dos cosas iguales a una tercera son iguales entre sí.

2.- Si cantidades iguales se suman a cantidades iguales, las sumas son iguales.

3.- Si cantidades iguales se restan de cantidades iguales, las diferencias son iguales.

4.-Dos figuras que coinciden son iguales entre sí.

5.- El todo es mayor que cualquiera de sus partes.

E\&A

IIES

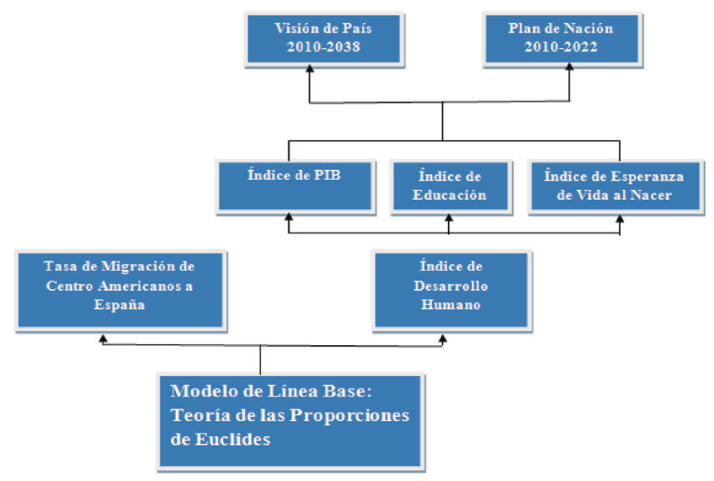

Figura 1.

Mapa Conceptual del Modelo de Línea Base de la Investigación

Fuente: Interpretación Propia del Modelo

\section{¿Por qué tomar a España como referencia de los migrantes Centro Americanos?}

Si analizamos la Figura 1 y el Gráfico No. 2, observamos que la mayor parte de los migrantes en España, son procedentes de Iberoamérica, señalando que el ritmo al cual estos migran hacia esa localidad, es considerablemente acelerado (INE, 2012).

8 http://www.oya-es.net/reportajes/euclides.htm 


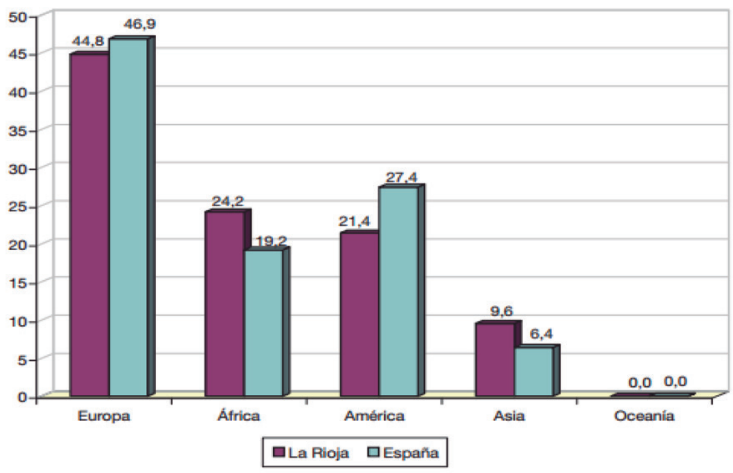

Gráfico No. 2.

\section{Composición de la Población Extranjera en España y la Rioja año 2012}

Fuente: INE Padrón municipal de Habitantes España 2012

Tabla No. 1

Proporción de Extranjeros en España en el tiempo (2000-2010)

\begin{tabular}{|l|r|r|r|r|r|r|r|}
\cline { 2 - 9 } \multicolumn{1}{c|}{} & \multicolumn{3}{c|}{ Población en Padrón } & \multicolumn{4}{c|}{ Extranjeros en Padrón } \\
\cline { 2 - 9 } \multicolumn{1}{c|}{} & TOTAL & \multicolumn{1}{c|}{ Incrent $^{\circ}$ anual } & $\%$ /TOTAL & \multicolumn{1}{c|}{ TOTAL } & \multicolumn{2}{c|}{ Incrent $^{\circ}$ anual } \\
\hline ene-00 & 40.499 .791 & & & $2,28 \%$ & 923.879 & & \\
\hline ene-01 & 41.116 .842 & 617.051 & $1,52 \%$ & $3,33 \%$ & 1.370 .657 & 446.778 & $48,36 \%$ \\
\hline ene-02 & 41.837 .894 & 721.052 & $1,75 \%$ & $4,72 \%$ & 1.972 .946 & 602.289 & $43,94 \%$ \\
\hline ene-03 & 42.717 .064 & 879.170 & $12,10 \%$ & $6,24 \%$ & 2.664 .168 & 691.222 & $35.04 \%$ \\
\hline ene-04 & 43.197 .684 & 480.620 & $1,13 \%$ & $7,02 \%$ & 3.034 .326 & 370.158 & $13,89 \%$ \\
\hline ene-05 & 43.975 .375 & 777.691 & $1,80 \%$ & $8,48 \%$ & 3.730 .610 & 696.284 & $22,95 \%$ \\
\hline ene-06 & 44.709 .964 & 733.589 & $1,67 \%$ & $9,27 \%$ & 4.144 .466 & 413.856 & $11,09 \%$ \\
\hline ene-07 & 45.200 .737 & 491.773 & $1,10 \%$ & $10,00 \%$ & 4.519 .554 & 375.088 & $9,05 \%$ \\
\hline ene-08 & 46.157 .822 & 957.085 & $2,12 \%$ & $11,41 \%$ & 5.268 .762 & 749.208 & $16,85 \%$ \\
\hline ene-09 & 46.745 .807 & 587.985 & $1,27 \%$ & $12,08 \%$ & 5.648 .671 & 379.909 & $7,21 \%$ \\
\hline ene-10 & 46.951 .532 & 205.725 & $0,44 \%$ & $12,16 \%$ & 5.708 .940 & 60.269 & $1,07 \%$ \\
\hline
\end{tabular}

Fuente: http://www.enlaceacademico.org/uploads/media/MIGRANTESESPANA.pdf

Por tanto se ha tomado a la República Española, como referencia migratoria en Europa.

Otros factores que justifican la selección de España como punto de quiebre de la migración latinoamericana, deriva a que el éxodo hacia Europa, ha llegado a tal grado que en España se han incrementado un sinnúmero de indicadores de riesgo migratorio, reflejado en el "Padrón 2000-2009”, los cuales presentan lo siguiente: 
- $\quad$ El 74,17\% del crecimiento de la población en España se debe a los extranjeros, que crecieron siempre por encima del 10\% año tras año entre 2000 y 2007.

- El año 2008, con un incremento del 7,21\%, parece iniciar un cambio de tendencia, confirmado en el año 2009 (incremento de 1,07\%), hacia la ralentización de la llegada de extranjeros.

- En 2008 Eurostat coloca a España en el sexto lugar de la UE de mayor porcentaje de población extranjera (12,3\%) por delante de países como Alemania, Francia y Reino Unido, que al hallarse en un estado más avanzado del ciclo inmigratorio tienen mayor número de nacionalizaciones (segunda y tercera generación).

- Al finalizar el año 2009 el 14,21\% de los empadronados es de origen extranjero. En la cohorte de la edad laboral (16-65 años), suponen el 12,08\% del total (INE, 2012).

\section{El Índice de Desarrollo Humano (IDH) y su cálculo:}

En este apartado se define el procedimiento, sobre el cual se basa el Índice de Desarrollo Humano, el cual desde su concepción (1996) ha sufrido evoluciones a lo largo de los años, sin embargo, la metodología y objetivo general de este, se mantiene.

E\&A

Cabe mencionar que este índice deriva del análisis de 3 variables "madres", las cuales poseen a su vez limites, inferiores y superiores, sobre los cuales, se aborda el cálculo de cada una de las propuestas de crecimiento o decrecimiento, del desarrollo humano, en sociedades en especificas. ${ }^{9}$

¿Qué es el IDH y cómo funciona?

El índice de Desarrollo Humano, es un indicador del desarrollo humano por país, elaborado por el Programa de las Naciones Unidas para el Desarrollo (PNUD). Se basa en un instrumento de medición socio-estadístico compuesto por tres parámetros: Esperanza de vida al nacer, salud, educación y nivel de vida digno $(\mathrm{PIB})^{10}$. Tomando en cuenta:

a. Longevidad (medida en función de la esperanza de vida al nacer).

b. Nivel educacional, medido en función de una combinación de alfabetización de adultos (ponderación, dos tercios) y tasa de matriculación (sic) combinada primaria, secundaria y terciaria (ponderación, un tercio).

c. Nivel de vida, medido por el PIB per cápita real (PPA en dólares).

9 http://www.eumed.net/libros/2008a/360/CALCULO\%20DEL\%20INDICE\%20DE\%20DESARROLLO\%20HUMANO\%20POR\%20 EL\%20PNUD.htm

10 http://es.wikipedia.org/wiki/\%C3\%8Dndice_de_desarrollo_humano 
Para el cálculo del índice, se han establecido valores mínimos y máximos fijos para cada uno de esos indicadores, como ser:

i. Esperanza de vida al nacer: 25 años y 85 años.

ii. Alfabetización de adultos: $0 \%$ y $100 \%$.

iii. Tasas de matriculación combinada: $0 \%$ y $100 \%$

iv. PIB per cápita real (PPA en dólares): PPA 100 dólares y PPA

Lo que nos lleva a la pregunta ¿Cómo se desarrolla el cálculo del IDH?

El cálculo del IDH, deriva de la articulación de las 3 variables antes mencionadas, con la incorporación de otros elementos, de corte académico, financiero y social, los cuales conforman la siguiente formula y estos son:

$\mathrm{IDH}=\frac{1}{3}(\mathrm{IEV})+\frac{1}{3}(\mathrm{IE})+\frac{1}{3}(\mathrm{IPBI})$

1. $\mathrm{IEV}=$ Índice de esperanza de vida

2. $\mathrm{IE}=$ Índice de educación

3. $\mathrm{IPIB}=$ Índice del PIB

\section{Cálculo del Índice de Esperanza de Vida:}

IEV Valor real - valor mínimo / Valor Máximo - Valor mínimo

\section{Cálculo del Índice de Educación.}

2/3 (Índice de Alfabetización) + 1/3 (Índice de Matriculación)

\section{Cálculo del Índice del PIB}

Log (Valor real) - Log (valor mínimo) / Log (Valor Máximo) - Log (Valor mínimo)

\section{CONSTRUCTO DEL MODELO}

Este diagrama sagital, nos permite apreciar, con claridad a las 3 variables que conforman el IDH, así como la relación sugerida por el objetivo general de la propuesta.

X1: Índice del PIB

X2: Índice Educación

X3: Índice de Esperanza de Vida al Nacer

X4: Índice de Desarrollo Humano

Y1: Tasa de Migración hacia España 


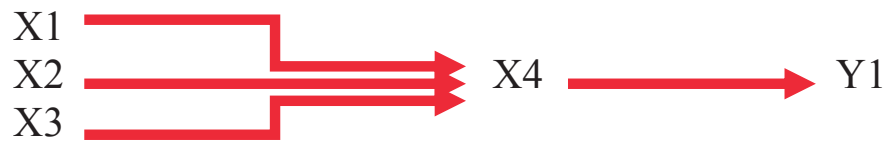

Figura No. 2.

Constructo del Modelo

Fuente: Interpretación propia

Donde la virtud del modelo radica en determinar cuál de las variables X1, X2 o X3, impacta de mayor manera sobre la variable X4 y a su vez, cuantificar el nivel o intensidad de la dependencia entre la variable X4 y Y1.

El análisis de la variable X4, permitirá explorar de forma causal, el origen de la fuga o migración de la población hondureña hacia el extranjero (España) bajo el enfoque del IDH. Posteriormente, se procederá a estudiar la relación e intensidad de la dependencia (si existe) entre IDH-Tasa de migración Centro Americana hacia a España.

\section{HIPÓTESIS DEL MODELO}

\section{Hipótesis No. 1:}

Ho:

La variable que más impacto genera, sobre el X4 (IDH) es la del X1 (IPIB).

H1:

La variable que más impacto genera, sobre el X4(IDH) es la del X2 (IE).

\section{Hipótesis No. 2:}

Ho:

El nivel de correlación entre las variables X4 (IDH)-Y1, oscila entre 0.85- 0.95 y si existe evidencia estadística que demuestre la dependencia entre las variables.

H1:

El nivel de correlación entre las variables X4 (IDH) -Y1, oscila entre 0.50-0.60 y no estadística que demuestre la dependencia entre las variables. 


\section{Hipótesis No. 3:}

Ho: Existe evidencia estadística que demuestre la dependencia entre las variables X2-Y1

H1: No existe evidencia estadística que demuestre la dependencia entre las variables X2-Y1

Para el desarrollo del apartado de validación de hipótesis, se efectuaran 2 procesos generales,

1. Obtención de los niveles de correlación entre dichas variables.

2. Desarrollo que conllevan a las pruebas t-student sobre los datos recabados (Hipótesis No. 3), los cuales contemplaran los siguientes pasos:
a. Análisis de Medias.
b. Análisis de Varianzas.
c. Interpretación de la evidencia estadística que demuestre que hay existe la relación y dependencia entre la las variables.

\section{Obtención de los niveles de correlación entre dichas variables.}

A continuación se presentaran las correlaciones entre las distintas combinaciones de variables, propuestas en el constructo del modelo, consolidada en la Tabla No. 2.

Tabla No. 2

Nivel de correlación entre las variables del constructo

\begin{tabular}{|l|l|l|l|l|}
\hline & \multicolumn{1}{|c|}{$\begin{array}{c}\text { Nivel de Correlación } \\
\text { IPIB-IDH por País }\end{array}$} & $\begin{array}{c}\text { Nivel de } \\
\text { Correlación IE- } \\
\text { IDH por País }\end{array}$ & $\begin{array}{c}\text { Nivel de } \\
\text { Correlación IEV- } \\
\text { IDH por País } \\
\text { Correlación } \\
\text { Tasa Migración- } \\
\text { IDH por País }\end{array}$ \\
\hline HONDURAS & 0.993741608 & 0.99909861 & 0.993652256 & 0.933854935 \\
\hline COSTA RICA & 0.985335108 & 0.99437109 & 0.980497488 & 0.913974318 \\
\hline GUATEMALA & 0.974696023 & 0.99636403 & 0.997217274 & 0.911456191 \\
\hline EL SALVADOR & 0.99260985 & 0.99627994 & 0.968715973 & 0.868779454 \\
\hline NICARAGUA & 0.905990127 & 0.99463241 & 0.988660296 & 0.93165927 \\
\hline PANAMA & 0.966488101 & 0.98262233 & 0.997871799 & 0.916917434 \\
\hline$\sum$ niveles: & 5.818860817 & $\mathbf{5 . 9 6 3 3 6 8 4 2}$ & 5.926615085 & $\mathbf{5 . 4 7 6 6 4 1 6 0 2}$ \\
\hline
\end{tabular}

Fuente: Cálculos e Interpretación propia, de la fuente: http://hdr.undp.org/es/datos/tendencias/ 
Tras evaluar los niveles de dependencia entre las 3 variables que conforman el IDH, podemos citar, que a nivel de Centro América, la correlación entre la relación Índice de Educación - Índice de Desarrollo Humano presentaron una mayor intensidad que las 2 combinaciones restantes (IEV-IDH, IPIB-IDH).

Otro punto a resaltar en nuestro análisis, parte de la relación Tasa de Migración - IDH, la cual demostró poseer un nivel de correlación aceptable (a nivel de Centro América), con un valor de $\mu=0.912$.

Conclusiones sobre las Hipótesis No. 1,2 y 3

Tabla No. 3

Resumen de las Conclusiones, proceso de validación de Hipótesis

\begin{tabular}{|c|c|c|c|c|}
\hline $\begin{array}{c}\text { Tipo de } \\
\text { Hipótesis }\end{array}$ & $\begin{array}{c}\text { Niveles de } \\
\text { Correlación }\end{array}$ & $\begin{array}{c}\text { Análisis de } \\
\text { Medias }\end{array}$ & $\begin{array}{c}\text { Análisis de } \\
\text { Varianza }\end{array}$ & Hipótesis Aceptada \\
\hline Hipótesis No. 1 & $\begin{array}{l}0.993 \text { nivel de } \\
\text { Correlación IE-IDH, } \\
\text { por tanto se acepta la } \\
\text { hipótesis alternativa } \\
\text { H1 }\end{array}$ & ------- & ------- & \\
\hline Hipótesis No. 2 & $\begin{array}{l}0.913, \quad \text { media } \\
\text { ponderada de la } \\
\text { correlación entre } \\
\text { variables a nivel de } \\
\text { Centro América }\end{array}$ & $\begin{array}{l}\text { Sig.: } 0 \text {, por tanto } \\
\text { no hay evidencia } \\
\text { es t a dis t i c a } \\
\text { que valide la } \\
\text { relación entre } \\
\text { estas variables }\end{array}$ & $\begin{array}{l}\text { Sig.: } 1.7973 E- \\
06 \text { por tanto no } \\
\text { hay suficiente } \\
e v i d \text { e } n \text { c } i \text { a } \\
\text { estadistica que } \\
\text { valide la relación } \\
\text { entre las variables }\end{array}$ & $\begin{array}{l}\text { Se acepta la Hipótesis } \\
\text { Alternativa H1, no } \\
\text { hay relación entre } \\
\text { las variables Tasa de } \\
\text { Migración - Índice de } \\
\text { Desarrollo Humano }\end{array}$ \\
\hline Hipótesis No. 3 & $\begin{array}{l}0.953, \quad \text { media } \\
\text { ponderada de la } \\
\text { correlación entre } \\
\text { variables a nivel de } \\
\text { Centro América }\end{array}$ & $\begin{array}{l}\text { Sig.: } 0 \text {, por tanto } \\
\text { no hay evidencia } \\
\text { es t a dis tic a } \\
\text { que valide la } \\
\text { relación entre } \\
\text { estas variables }\end{array}$ & $\begin{array}{l}\text { Sig.: } 0.00254711 \\
\text { por tanto no } \\
\text { hay suficiente } \\
e v i d \text { e c } i \text { a } \\
\text { estadistica que } \\
\text { valide la relación } \\
\text { entre las variables }\end{array}$ & $\begin{array}{l}\text { Se acepta la Hipótesis } \\
\text { Alternativa H1, no } \\
\text { hay relación entre } \\
\text { las variables Tasa de } \\
\text { Migración - Índice de } \\
\text { Educación }\end{array}$ \\
\hline
\end{tabular}

Fuente: Interpretación propia

Si repasamos el constructo (Figura No. 2), podemos observar las siguientes relaciones:

$\mathrm{X} 1$ (Índice del PIB) X4 (Índice de Desarrollo Humano)

X2 ( Índice Educación) X4 (Índice de Desarrollo Humano) X3 (Índice de Esperanza de Vida al Nacer) $\longrightarrow$ X4 (Índice de Desarrollo Humano) X4 (Índice de Desarrollo Humano) D Y1 (Tasa de Migración) 
La hipótesis No. 1 nos muestra que realmente existe una alta correlación entre las variables IE-IDH, de acuerdo a una media ponderada obtenida de todos los coeficientes de correlación, para los países en estudio, a nivel de Centro América (para la relación II de la Figura No. 2), de igual forma, tras evaluar los coeficientes de correlación de las demás combinaciones (I y III) en la Tabla No. 3, de este documento, podemos observar que en efecto, el componente que más incide sobre el IDH a nivel de Centro América, es el Índice de Educación, por tanto se acepta la hipótesis alternativa H1.

El abordaje de la hipótesis No. 2, sugiere de forma paralela al análisis de correlación entre las relaciones I, II, III y IV, la incorporación de una prueba t-student para corroborar la existencia de evidencia estadística que valide la dependencia entre las variables de la relación IV.

Si observamos la Tabla No. 3 y el abordaje de las pruebas de medias y varianzas, podemos inferir que no hay evidencia empírica que demuestre la dependencia entre las variables Índice de Desarrollo Humano e Tasa de Migración, aceptando la Hipótesis Alternativa H1.

De igual forma, si evaluamos la hipótesis No. 3, podemos observar que tiene sentido el desarrollo de

la Hipótesis No. 2, puesto que la relación II, depende estrictamente de la relación IV y si la relación IV, no se cumple, la II de igual forma no se cumplirá.

Es en este punto de hacemos uso de la ley de la proporcionalidad de Euclides la cual cita lo siguiente:

Si D depende de X

Si A depende de D

Si B depende de D

y

Si C depende de D

Entonces si D no depende de X,

$\mathrm{Ni} \mathrm{A}$, ni $\mathrm{B}$, ni C tendrán relación/vínculo con $\mathrm{X}$ 
De igual forma este axioma, formulada por Euclides, aplicado en la resolución de la Hipótesis sin embargo, siempre se efectuaron los análisis de medias y varianza (para la Hipótesis No. 3), los cuales corroboraron el cumplimiento de esta teoría y demostraron que nos hay evidencia estadística que valide la existencia de que exista dicha relación.

Destacando, entre las distintas limitantes para la realización de este análisis:

- La no existencia de un flujo migratorio correspondiente a la región Centro Americana hacia España, particularmente la hondureña.

- La no existencia información respecto de las caídas o alzas de la movilización poblacional Centro Americana en España.

- La no existencia de indicadores que soporten los niveles académicos de aquellos migrantes Centro Americanos en España.

Finalmente, el apartado que más trasciende sobre el Índice de Desarrollo Humano, es el Índice de Educación, de igual forma, los análisis estadísticos demuestran que el ritmo sobre el cual se gesta la migración centroamericana hacia Europa, no es proporcional a la relación con la cual la caída de las condiciones sociales de desarrollo poblacional se gestan.

\section{REFERENCIAS}

Cejas, A. M. (2002). Teoria de las Proporciones de Euclides. Tenerife: Universidad de la Laguna, España.

Dijkstra, G. (2007). Evaluación de las Estrategias de Reducción . En J. R. Niek de Jong, Honduras: ¿Qué pasó con la ERP? . Holanda, Rotterdam: Universidad de Rotterdam.

INE, E. (2012). Extranjeros en la UE y en España. Madrid: Cifras INE Boletin informativo. Terragno, R. (17 de Octubre de 2010). El Gran Brasil no empezó ni terminará con Lula. El Clarin, págs. 5-6. 